EPJ manuscript No.

(will be inserted by the editor)

\title{
Non-Valence Fock States in Heavy-to-Light Form Factors at Large Recoil
}

Björn O. Lange

CLNS $03 / 1845$

Newman Laboratory for Elementary-Particle Physics, Cornell University, Ithaca, NY 14853, U.S.A.

Received: date / Revised version: date

\begin{abstract}
We studied three-particle Fock state contributions to heavy-to-light form factors in the context of soft-collinear effective theory and found that they enter at leading power. These contributions are nonfactorizable due to the appearance of endpoint singularities, however they do not violate spin-symmetry relations at leading power. In this talk I present their numerical estimation in a crude model in which the "soft overlap" contribution is cut off and find that they might lower the standard values for the form factors at maximum recoil significantly. Furthermore I briefly discuss the role of soft-collinear messenger modes in the region of soft overlap.
\end{abstract}

PACS. 12.39.St Factorization - 13.20.He Decays of bottom mesons

\section{Introduction}

The calculation of many $B$-decay amplitudes can be significantly simplified using QCD factorization theorems [1, and soft-collinear effective theory (SCET) 2] provides an excellent environment to discuss such factorization proofs. It is of great interest to apply this technology to $B \rightarrow$ light meson form factors, since they enter the calculation of amplitudes for many important rare decays such as $B \rightarrow K^{*} \gamma, B \rightarrow \pi \pi$, etc. At large recoil the leading contributions to the 10 standard form factors can be described by only three independent universal functions $\zeta_{p}, \zeta_{\|}, \zeta_{\perp}$, due to the spin-symmetry of highly energetic fermions [3]. These relationships are, however, not exact, and an interesting question is whether corrections to them can be written in a factorized form, as proposed by Beneke and Feldmann 4,5]

$$
f_{i}\left(q^{2}\right)=C_{i} \zeta(E)+\phi_{B} \otimes T_{i} \otimes \phi_{L}+\ldots
$$

Here, $\phi_{B}$ and $\phi_{L}$ denote leading order light-cone distribution amplitudes (LCDAs) of the $B$ meson and the light recoiling meson, respectively. The coefficients $C_{i}$ and the kernel functions $T_{i}$ are perturbatively calculable and the ellipses denote power suppressed contributions. The symbols $\otimes$ denote a convolution integral, which emerges through spectator interactions. Note that this formula does not define the functions $\zeta(E)$ unambiguously and that factorizable contributions to the form factor $f_{i}$ can be associated with either term in (10) as long as they obey the spin-symmetry relations.

Part of any proof of factorization must address the question of convergence of the convolution integrals, as well as the question whether two-particle Fock states dom- inate the amplitude at leading power. The main motivation for our study was to find examples in which this is not the case or for which answers to these questions are non-trivial.

In this talk I discuss the role of three-particle Fock state contributions to the form factors [6]. They are found to appear in leading power and are non-factorizable due to endpoint singularities. However, they do not violate spinsymmetry and can therefore be associated with the first term in equation (1).

\section{Calculation}

The goal of this calculation is to match the QCD amplitude $\left\langle L\left|\bar{\psi}_{q} \Gamma \psi_{b}\right| B\right\rangle$, where $L$ represents a light pseudoscalar or vector meson and $\Gamma$ an appropriate Dirac structure, onto the corresponding SCET operator matrix elements. These operators contain at least one heavy quark and one light soft quark in the initial state and two collinear quarks in the final state. We calculated three different classes of diagrams that include spectator interactions: the two-particle to two-particle amplitude, as shown in Fig. [1 the two-particle to three-particle amplitude, in which the operators contain one extra final collinear gluon, and
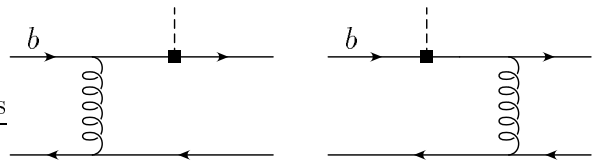

Fig. 1. Spectator interaction via exchange of a hard-collinear gluon. 
Björn O. Lange: Non-Valence Fock States in Heavy-to-Light Form Factors at Large Recoil

the three-particle to two-particle amplitude with one extra soft gluon in the initial state. We obtain contributions to the matching calculation by attaching an extra gluon anywhere in either of the two QCD diagrams in Fig. 11as long as this leads to an additional off-shell propagator. These off-shell modes are either hard or hard-collinear and will be integrated out in the matching procedure. Diagrams that involve emission of an extra collinear gluon from the heavy quark line lead to power suppression; however, the remaining diagrams yield leading power contributions to the form factors, which then involve three-particle Fock state LCDAs of the final light meson after projecting onto the physical states. The same can be observed for diagrams involving three partons of the $B$ meson. The results for each of the three classes include terms that diverge logarithmically at the endpoints, such as, for example,

$$
\int_{0}^{\infty} d \omega \frac{\phi_{+}^{B}(\omega)}{\omega^{2}} \text { or } \quad \int_{0}^{1} d x \frac{\phi_{\pi}(x)}{(1-x)^{2}} .
$$

It is convenient to introduce light-cone coordinates, in which case $\omega$ can be identified with the "plus-component" of the soft spectator momentum, and $x$ denotes the fraction of the "minus-component" of the pion momentum carried by the collinear quark emerging from the weak interaction. The integrals in (2) diverge as $\omega$ or $\bar{x}=1-x$ become smaller than the originally assigned scaling in powers of $\lambda=\Lambda / m_{b}$ in SCET. For comments see section 4

Let me stress here that these endpoint singularities arise in a direct matching of $Q C D \rightarrow S C E T$. In a two-step matching procedure $Q C D \rightarrow S C E T_{I} \rightarrow S C E T_{I I}$, where the intermediate theory lives on a hybrid scale $\sqrt{\Lambda m_{b}}$ and the final theory coincides with $S C E T$, it has been argued 7] that no divergences appear in the first matching step. We must therefore conclude that the second matching step is non-trivial in that factorizable terms in $S C E T_{I}$ do not necessarily match onto factorizable terms in $S C E T_{I I}$.

\section{A rough numerical estimation}

Another interesting aspect of our analysis arises from the fact that three-particle contributions to the final light meson appear at leading power. In this section we would like to estimate their relative numerical impact on the three universal form factors, which we define, in accordance with [4], to coincide with the standard form factors

$$
\zeta_{P}=f_{+} \quad, \quad \zeta_{\|}=A_{0} \quad, \quad \zeta_{\perp}=T_{1} .
$$

We regularize divergent integrals, such as (2), by introducing cutoffs $\omega_{0}=\epsilon_{h} \lambda_{B}, x_{0}=\epsilon_{l}$ which separate the contributions near ("soft overlap") and away from the endpoints. The HQET parameter $1 / \lambda_{B}$ is defined as the first inverse moment of $\phi_{+}^{B}$ and provides the necessary mass dimension to $\omega_{0}$. Our belief is that the situation near the endpoints can not be correctly described by soft and collinear degrees of freedom only, but rather necessitates the introduction of a "soft-collinear" mode 11,12. The
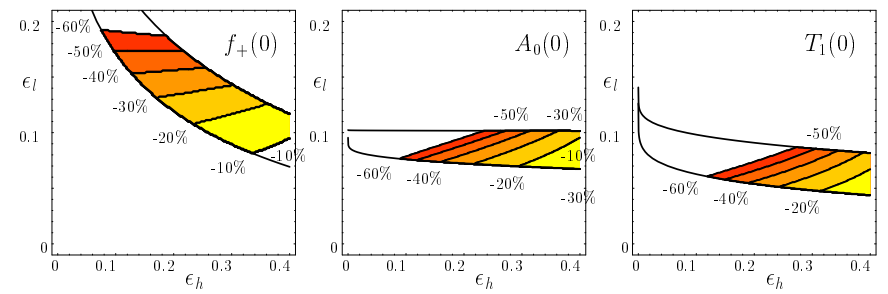

Fig. 2. Visualization of the relative size of three-particle Fock state contributions to the form factors $f_{+}(0), A_{0}(0)$ and $T_{1}(0)$. The bands reflect the regions in parameter space in which all terms that cannot be associated with a three-particle configuration of the light final meson give rise to the values found by LCSR within a $30 \%$ error. These bands are color-coded by the value of the ratio of three-particle and two-particle contributions.

discussion of their role is left for section 4. Here we simply set these contributions to zero and study the dependence of the regulated convolution integrals to the cutoffs $\epsilon_{l}$ and $\epsilon_{h}$, keeping in mind that these cutoffs serve as a transition mark between soft $(\omega \sim \lambda)$ and soft-collinear modes $\left(\omega \sim \lambda^{2}\right)$, or collinear $(\bar{x} \sim 1)$ and soft-collinear $(\bar{x} \sim \lambda)$ modes. Typical values of these cutoffs are around $\left(\epsilon_{l}, \epsilon_{h}\right) \approx(0.1,0.3)$.

Since we would like to compare relative contributions from three-particle configurations of the final light meson we define two quantities for each form factor: $\triangle F_{i}(2 p)$ denotes all terms involving purely two-particle final states, i.e. in the pseudoscalar case $\phi_{\pi}$ and the two-particle part of the twist-three wave-function $\phi_{p}$. Correspondingly $\triangle F_{i}(3 p)$ collects terms of three-particle origin. In Fig. 2 we show regions in the $\epsilon_{l}-\epsilon_{h}$ plane in which the $\triangle F_{i}(2 p)$ correspond to values predicted by light-cone sum rules (LCSR) [8 . 9 . This is reasonable since the LCSR analysis finds a negligible numerical impact from three-particle configurations, quoting [8]: "putting all intrinsic higher-twist parameters [...] to zero, the form factors change by at most 3\%". The band width denotes a generous $30 \%$ error on the LCSR central values. We color-coded these bands with the ratio $\triangle F_{i}(3 p) / \triangle F_{i}(2 p)$.

The immediate observation is that the three-particle contributions are negative for all reasonable values of $\left(\epsilon_{l}, \epsilon_{h}\right)$ and hence lower the numerical values for the form factors. Furthermore their impact is significant in the vicinity of $\left(\epsilon_{l}, \epsilon_{h}\right) \approx(0.1,0.3)$ and might be, with the exception of $f_{+}$, as large as $50 \%$.

Although the above model serves only as a very crude estimate, one must wonder how this result compares with the method of LCSR. The main difference between the Factorization/SCET approach and the LCSR approach lies in the sensitivity to the light-cone structure of the $B$ meson through heavy-to-light currents that are smeared over the light-cone in the former case and the interpolation of the $B$ meson by local currents in the latter. Furthermore the continuum model adopted in LCSR effectively cuts off any contributions near the endpoints $\bar{x} \rightarrow 0$ in a rough way. The question whether the method of LCSR reliably captures the effects of spectator interactions has 
recently been addressed in 10. In this work it was pointed out that one can probe the light-cone structure of the $B$ meson by invoking a second sum rule for the (process independent) HQET parameter $1 / \lambda_{B}$, albeit missing finer (process dependent) details related to spectator interactions.

\section{Endpoint configurations}

The existence of endpoint singularities suggests that the phase-space below the cutoffs $\epsilon_{l}$ or $\epsilon_{h}$, the soft-overlap region, obtains no suppression. If we describe the soft spectator momentum in light-cone coordinates it scales like $(\lambda, \lambda, \lambda)$, whereas the collinear momentum scales like $\left(\lambda^{2}, 1, \lambda\right)$. However, considering the situation in which $\omega$ becomes smaller, i.e. below the cutoff, the soft mode naively changes into $(\lambda, \lambda, \lambda) \rightarrow\left(\lambda^{2}, \lambda, \lambda\right)$. Similarly, in the endpoint $\bar{x} \rightarrow 0$ the collinear scaling changes into $\left(\lambda^{2}, 1, \lambda\right) \rightarrow$ $\left(\lambda^{2}, \lambda, \lambda\right)$. In SCET, these configurations are described by on-shell fields whose momenta scale like $\left(\lambda^{2}, \lambda, \lambda^{3 / 2}\right)$. This mode, called "soft-collinear mode", has recently been studied in 11 12 using the method of regions. In the case of form factors we can construct box and pentagon diagrams and show that soft-collinear exchanges lead to leading loop-momentum regions.

Note that in this region of phase-space the formally hard-collinear gluon interacting with the spectator now becomes soft or collinear, and is thus no longer integrated out when matched onto SCET operators. Since we can choose the external states to be eigenstates of the leading order SCET Lagrangian we may allow for one more unsuppressed soft or collinear gluon exchange between the upper and lower fermion line in Fig. 11 to form a box or pentagon diagram with only soft or collinear external legs.

However, there is no need to keep these soft-collinear modes in the theory, because we do not allow for such external momenta and thus require no source terms in the path integral. It is therefore possible to integrate them out, leaving an induced non-local interaction between soft and collinear fields which symbolically takes the form

$$
S_{S C}=\int d^{4} x d^{4} y \frac{\triangle\left(x_{-} y_{+}\right)}{\left(x_{-} y_{+}\right)^{2}} \bar{X} \mathcal{A}_{c}\left[x_{-}\right] \mathcal{A}_{s} \mathcal{Q}\left[y_{+}\right] .
$$

The explicit form can be found in [1]. In this notation $\mathcal{X}$ and $\mathcal{A}_{c}$ denote gauge invariant collinear quark and gluon fields, $\mathcal{Q}$ and $\mathcal{A}_{s}$ denote gauge invariant soft quark and gluon fields [13, and $\triangle$ is a soft-collinear propagation function. Power counting shows that this interaction scales like $\lambda^{3 / 2}$ and therefore enters the form factor calculation at leading power 6 .

Let me summarize: at leading power in SCET we find four types of operators which appear in the tree-level matching of QCD heavy-to-light form factors. The first three types are "local" operators (except for a light-cone separation) containing two+two, three+two and two+three soft + collinear fields. In the soft overlap region we have in addition to them a time-ordered product of a heavy-tocollinear current with the induced soft-collinear interaction (4).

\section{Conclusion}

Heavy-to-light form factors at large momentum transfer are terrific (and terrifying) examples for processes in which factorization is problematic. Two- and three-particle Fock states contribute at leading power and display endpoint singularities. We studied the first effect numerically by disregarding the soft overlap and introducing cutoff regulators that render the convolution integrals finite. It was found that three-particle contributions might lower the numerical values of the form factors significantly, especially in the $B \rightarrow V$ case, which is also favored by the analysis of radiative decays $B \rightarrow V \gamma$ in the framework of factorization 14,15. Our calculation also supports the finding by Luo and Rosner [16] who extrapolated the $q^{2}$ dependence of pseudoscalar form factors from lattice data into the low $q^{2}$ region and favor rather low values . Lastly I discussed soft-collinear messenger modes and endpoint singularities.

\section{Acknowledgments}

This work has been performed in collaboration with Farrukh Chishtie and Matthias Neubert, to whom I am greatly indebted. I also would like to thank M. Beneke, T. Feldmann, S. Bosch, E. Lunghi and S. Descotes-Genon for many interesting discussions during this conference.

\section{References}

1. M. Beneke et al., Phys. Rev. Lett. 83, 1914 (1999); Nucl. Phys. B 591, 313 (2000).

2. C. W. Bauer, S. Fleming and M. E. Luke, Phys. Rev. D 63, 014006 (2001); C. W. Bauer et. al., Phys. Rev. D 63, 114020 (2001); C. W. Bauer and I. W. Stewart, Phys. Lett. B 516, 134 (2001); C. W. Bauer, D. Pirjol and I. W. Stewart, Phys. Rev. D 65, 054022 (2002); M. Beneke et. al., Nucl. Phys. B 643, 431 (2002).

3. J. Charles et. al., Phys. Rev. D 60, 014001 (1999).

4. M. Beneke and T. Feldmann, Nucl. Phys. B 592, 3 (2001).

5. M. Beneke and T. Feldmann, this conference, also hep-ph/0308303.

6. F. Chishtie, B. O. Lange and M. Neubert, in preparation.

7. C. W. Bauer, D. Pirjol and I. W. Stewart, hep-ph/0211069.

8. P. Ball and V. M. Braun, Phys. Rev. D 58, 094016 (1998).

9. P. Ball, JHEP 9809, 005 (1998); P. Ball and R. Zwicky, JHEP 0110, 019 (2001).

10. P. Ball, hep-ph/0308249.

11. T. Becher, R. J. Hill and M. Neubert, hep-ph/0308122.

12. T. Becher, R. J. Hill, B. O. Lange and M. Neubert, hep-ph/0309227.

13. R. J. Hill and M. Neubert, Nucl. Phys. B 657, 229 (2003).

14. S. W. Bosch and G. Buchalla, Nucl. Phys. B 621, 459 (2002).

15. M. Beneke, T. Feldmann and D. Seidel, Nucl. Phys. B 612, 25 (2001).

16. Z. Luo and J. L. Rosner, hep-ph/0305262. 